\title{
Multiplierless Implementation of Rotators and FFTs
}

\author{
Malcolm D. Macleod \\ QinetiQ Ltd., St. Andrews Road, Malvern, Worcestershire WR14 3PS, UK \\ Email:mamacleod@iee.org \\ Received 9 December 2004; Revised 26 June 2005; Recommended for Publication by Markus Rupp
}

\begin{abstract}
Complex rotators are used in many important signal processing applications, including Cooley-Tukey and split-radix FFT algorithms. This paper presents methods for designing multiplierless implementations of fixed-point rotators and FFTs, in which multiplications are replaced by additions, subtractions, and shifts. These methods minimise the adder-cost (the number of additions and subtractions), while achieving a specified level of accuracy. FFT designs based on multiplierless rotators are compared with designs based on the multiplierless implementation of DFT matrix multiplication. These techniques make possible VLSI implementations of rotators and FFTs which could achieve very high speed and/or power efficiency. The methods can be used to provide any chosen accuracy; examples are presented for 12 to 26 bit accuracy. On average, rotators are shown to be implementable using 10,12, or 15 adders to achieve accuracies of 12,16 , or 20 bits, respectively.
\end{abstract}

Keywords and phrases: FFT implementation, rotator implementation, multiplierless design, VLSI.

\section{INTRODUCTION}

Complex rotators, which multiply input values by $e^{j \theta}$ for some $\theta$, are used in many important applications, including fast fourier transform (FFT) algorithms, where they are also known as "twiddle factors" [1]. Many current systems require embedded FFTs, including orthogonal frequencydivision multiplexing modems for digital broadcasting, wireless networking, and telecommunications, and many more potential applications are anticipated.

Because the real and imaginary parts of $e^{j \theta}$ are in general irrational, the computation of such rotations, and of the FFT, is inherently inexact [1], so the requirement is always to achieve sufficient accuracy for an intended application. To reduce power consumption and increase speed, fixed-point arithmetic is often used.

Until recently, research into implementation of these functions has concentrated on architectures such as programmable DSP ICs, containing multiplier-accumulators. With recent advances in VLSI technology, "multiplierless" algorithms now provide the option of further lowering power consumption and IC area, or greatly increasing throughput.

In multiplierless algorithms, general-purpose multipliers are replaced by binary shifts, adders, subtracters, negaters, and stores. As is common when considering VLSI hardware implementations, binary shifts and data moves are treated as costless, while stores and negaters are assumed to

This is an open access article distributed under the Creative Commons Attribution License, which permits unrestricted use, distribution, and reproduction in any medium, provided the original work is properly cited. be significantly less costly (in area or power consumption) than adders. Therefore subtracters are assumed to have the same cost as adders [2], and the measure of the implementation cost which is used is the "adder-cost", which is the total number of subtracters and adders.

Techniques have been developed for the minimumadder-cost implementation of individual multiplications [2, $3,4]$, digital filters $[5,6,7]$, and matrix multiplications $[7,8,9]$, including the DFT matrix [8].

Methods have also been described for designing multiplierless DCTs [10, 11], and for multiplierless implemention of the Winograd and prime-factor Fourier transform algorithms [12].

This paper describes methods for the design of minimum-adder-cost multiplierless rotators, and of CooleyTukey FFTs and related transforms such as the split-radix FFT. FFT designs based on multiplierless rotators are also compared with designs based on the multiplierless implementation of DFT matrix multiplication.

Rotators are complex multiplications by $w=c+j s$, where $s=\sin \theta$ and $c=\cos \theta$. If $\theta=k \pi / 2$, for $k$ integer, then implementation of the rotation is trivial (i.e., does not require any adders). Otherwise both $c$ and $s$ have magnitude less than one, so if they are represented as fixed-point two'scomplement values, they require only one (sign) bit before the binary point, and $b$ bits after it, where $b+1$ is the chosen wordlength. Multiplication by such a fixed-point value $c$ is therefore equivalent to multiplication by the integer $2^{b} c$ followed by division by $2^{b}$; hence without loss of generality all multiplication coefficients may be assumed to be integers. 
This paper will show that many alternate implementation structures must be searched in order to minimise rotator adder-cost. This expanded search procedure and the resulting low-cost multiplierless designs for rotator and FFT implementation are the novel contributions of the paper.

\section{EXISTING MULTIPLIERLESS FFTS}

FFT algorithms have a multistage structure. An $N$-point radix- $M$ FFT consists of $\log _{M} N$ stages, each containing $(N / M) M$-point DFTs, alternating with stages consisting of complex rotators. Radix-2 and radix-4 FFTs are widely used, because 2- and 4-point DFTs contain only trivial multiplications by \pm 1 or $\pm j$, but other choices of radix, mixed-radix, or split-radix FFTs [13] are possible.

Despain [1] commented that in many applications a fixed phase offset, or an arbitrary fixed scaling, of all the FFT outputs is allowable, and can if necessary be compensated for later, at low cost. If any such scaling is used, the same scaling must be applied to all data passing through any given stage of the FFT.

Despain described a modified radix-4 16-point CooleyTukey FFT [1] in which low adder-cost was achieved by allowing a common phase offset and scale factor to be applied to all the FFT outputs.

Perera and Rayner [14] described radix-4 FFTs based on blocks, each equivalent to a 4 -point DFT and 4 rotations, but implemented as a $4 \times 4$ matrix multiplication, in which each multiplier coefficient was constrained to be a sum of powers of two (SOPOT) with at most 1 adder (i.e., either $\pm 2^{k}$ or $\left.\pm 2^{k} \pm 2^{m}\right)$.

A recent more general method [15] implements rotators in a conventional FFT structure, using a specific rotator structure together with optimised SOPOT coefficients, having a user-selectable maximum number of adders.

\section{ALGORITHMS FOR MULTIPLIERLESS DESIGN}

For individual constant multipliers, the use of canonic signed digit (CSD) representation requires on average 33\% fewer adders than those required by normal binary [2]. Structures with fewer adders than CSD can be found which use factored and other forms; for example, $45 x=(1+4) \times(1+8) x$ only requires 2 adders, whereas multiplication using the CSD form requires 3 . Such structures may be found using the exhaustive minimised adder graph (MAG) algorithm [3], applied to integer coefficients up to $2^{12}$ in [3], and extended to $2^{19}$ in [4], or suboptimal algorithms such as those in [2].

In applications where two or more products of the same input value are required simultaneously, such as transposedform digital filters, a multiplier block $[6,7,8,16]$ may be used, and the number of adders may then be reduced by sharing terms. For example, to produce $9 x, 45 x$, and $13 x$ simultaneously, we may generate $9 x=(8+1) x, 45 x=(4+1) \times 9 x$, and $13 x=9 x+4 x$, at a total cost of 3 adders. A dependencegraph algorithm for designing minimum-adder-cost multiplier blocks was introduced by Bull and Horrocks [17]. In such algorithms graph edges represent binary shifts and/or negation, and graph vertexes (nodes) represent adders. An improved algorithm, named "Bull and Horrocks modified" (BHM), was presented in [6], together with another algorithm named " $n$-dimensional reduced adder graph" (RAG$n$ ). Reduced-adder-cost multiplier blocks may also be designed using common subexpression elimination (CSE) methods, for example $[7,16]$.

CSE methods may also be used to design reduced-cost multiplierless matrix multiplications $[7,8]$, in which there may be common subexpressions not only across outputs (as in multiplier blocks) but also across inputs.

\section{ROTATOR IMPLEMENTATION OPTIONS}

A rotation is a multiplication by $w=c+j s$, where $s=\sin \theta$ and $c=\cos \theta$, with the result $u+j v=(c+j s)(x+j y)=$ $(c x-s y)+j(s x+c y)$. It can be computed

(i) directly, using four separate multipliers (two by $c$ and two by s) and two additions;

(ii) by using a multiplier block to compute $c x$ and $s x$ simultaneously, and another identical one to compute $c y$ and $s y$, followed by two additions;

(iii) as $c(x-y)+(c-s) y+j(s(x+y)+(c-s) y)$; this requires 3 multiplications (by $c, s$, and $(c-s))$ and 4 additions;

(iv) as $y(c-s)+(x-y) c+j(x(c+s)-(x-y) c)$; this requires 3 multiplications (by $c,(c+s)$, and $(c-s)$ ) and 3 additions;

(v) as $x(c-s)+(x-y) s+j(y(c+s)+(x-y) s)$; this requires 3 multiplications (by $s,(c+s)$, and $(c-s))$ and 3 additions;

(vi) as in [15] by factorising the matrix representation of the complex rotation,

$$
\begin{gathered}
{\left[\begin{array}{l}
u \\
v
\end{array}\right]=\left[\begin{array}{cc}
c & -s \\
s & c
\end{array}\right]\left[\begin{array}{l}
x \\
y
\end{array}\right],} \\
\text { as }\left[\begin{array}{cc}
c & -s \\
s & c
\end{array}\right]=\left[\begin{array}{cc}
1 & t \\
0 & -1
\end{array}\right]\left[\begin{array}{cc}
1 & 0 \\
-s & 1
\end{array}\right]\left[\begin{array}{ll}
1 & -t \\
0 & -1
\end{array}\right],
\end{gathered}
$$

where $s=\sin \theta$ and $t=\tan (\theta / 2)$; this requires 3 multiplications (one by $s$ and two by $t$ ) and 3 additions;

(vii) by noting that a rotation by angle $\theta$ can be implemented as successive rotations by angles $\phi$ and $(\theta-\phi)$, as in the CORDIC algorithm [18]; or

(viii) by treating the rotation as a matrix multiplication as shown in (1), to which matrix CSE methods $[7,8]$ can be directly applied.

Despain's designs [1] use several (but not all) of the above options.

For the rotator-type (vii) we limited the number of cascaded rotations to two, partly to reduce search time and also because the adder-cost overhead of using more than two rotators makes a low-cost solution less likely to occur.

For rotator types (iii), (iv), and (v), two quantisation options are possible. The first is to round $c, s, c+s$, and $c-s$ independently. However, the rounded versions of $c+s$ and $c-s$ may not equal the sum/difference of the rounded versions of $c$ and $s$. In that case, the gain and phase shift produced by the quantised structure may vary slightly with the argument of the input value. This may also happen for rotator type (vi). 
The second option, which we label (iii a), (iv a), or (v a), is to quantise $c \pm s$ to the sum or difference of the rounded values of $c$ and $s$. For these variants, the gain and phase shift are independent of input argument.

For the special case of rotations by odd multiples of $\pi / 4$, a simpler structure is possible because $c= \pm s$. First, $c x$ and $c y$ are computed, and then two further additions or subtractions produce the result.

\section{ROTATOR OPTIMISATION}

To design the multiplierless form of one of the rotator types described in Section 4, given a desired rotation angle, we multiply its coefficients by an integer scale factor $k$, rounding the results to integers, and then evaluate its accuracy and adder-cost. To find the minimum-adder-cost solution which achieves the required accuracy, a search is carried out over a range of values of $k$, and over all the rotator types described in Section 4. If the overall gain of the rotator is required to be unity, $k$ is restricted to be a positive power of two, so that the gain can be made unity by a simple shift. If the nonunity overall gain is acceptable, then $k$ is allowed to be any integer.

Before starting the search, the minimum-adder-cost solutions for individual multiplications by each positive integer coefficient value up to a chosen maximum are precomputed, using the algorithms in $[2,3]$, and stored.

For rotator types (i), (iii), (iv), (v), (vi) and (iii a), (iv a), ( $\mathrm{v}$ a), these precomputed individual multiplier designs are used, while for option (ii), which uses multiplier blocks, two multiplier-block design methods, BHM [6] and RAG- $n$ [6], are applied and the results are compared. For the matrix CSE approach (viii), the algorithm described in [8] was used.

The two-stage rotator option (vii) has to be searched differently, for efficiency. First, all possible rotators having integer real and imaginary coefficients $c$ and $s$ which are either positive powers of two (SOPOT-0) or the sum of two such values (SOPOT-1) were generated, up to a specified maximum (in this paper, the maximum was set to $2^{16}$ ). The restriction to SOPOT-1 coefficients and the limited maximum magnitude are arbitrary, but they limit search time and storage requirements.

Next, all possible cascade combinations of two of these rotators (with either the same or opposite signs of the rotation angle) are generated, and the resulting equivalent complex multiplication coefficient of the combined rotator, $c_{e}+$ $j s_{e}$, is stored, along with its adder-cost.

Then, during the search phase, for a given scale factor $k$, each of the stored coefficients $c_{e}+j s_{e}$ in turn is multiplied by whichever integer power of two, $2^{K}$, makes the resulting coefficient magnitude $\left(2^{K} \sqrt{c_{e}^{2}+s_{e}^{2}}\right)$ closest to $k$, and the resulting error and cost are evaluated.

\subsection{Accuracy measurement}

The root-mean-square (RMS) error due to rounding random coefficients to binary fixed-point values with $b$ bits after the binary point is $2^{-b} / \sqrt{12}$. Consider a set of two or more actual coefficients, and let their actual RMS error be $\sigma_{C}$; for example, for a rotator, the actual (rounded) coefficients might be given by $c_{Q}=\operatorname{round}(k c)$ and $s_{Q}=\operatorname{round}(k s)$, and then $\sigma_{C}=\sqrt{\left(c_{Q} / k-c\right)^{2}+\left(s_{Q} / k-s\right)^{2}}$. We define the accuracy of such a set of coefficients as

$$
\hat{b}=-\log _{2}\left(\sqrt{12} \sigma_{C}\right) \text { bits. }
$$

Using this definition, a set of coefficients quantised with $b$ bits after the binary point will give an accuracy $\hat{b}$ from (3) which is close to $b$ bits. This allows a direct comparison between the accuracy actually achieved in a given case and that which one would expect to achieve by rounding coefficients to a given wordlength.

Despain [1] defined a term "precision", also measured in bits, which measures only the angular error, $\Delta \theta$, of a rotator and is given by $\log _{2}(2 \pi / \Delta \theta)$; these "precision" values are $2.5-3.3$ bits greater than the corresponding "accuracy" values given by (3).

For rotator types (i), (ii), (iii a), (iv a), (v a), and (viii), the actual multiplication coefficient of the rotator is that obtained by quantising the values of $c$ and $s$. For rotator type (vii), the effective coefficient is in general different. For rotator types (iii), (iv), (v), and (vi), the gain and phase shift produced by the quantised structure may vary slightly with the argument of the input value, therefore to compute the effective coefficient and accuracy of the rotator we compute the gain and mean-squared error over all input arguments. It is straightforward to show that this error is a periodic function of the input argument, with period $\pi / 2$. Hence the effective coefficient and the squared error are computed over a uniformly-spaced set of input arguments in the range $0 \cdots \pi / 2$, and the resulting mean-squared error is then used in (3).

\subsection{Results}

To demonstrate the results achievable by this approach, we designed rotators for the set of rotation arguments $p 2 \pi / 1024$, $p=1 \cdots 128$, using 3 scale factors, $k=2^{12}, 2^{15}$, and $2^{18}$; this leads to accuracies of approximately 12,15 , and 18 bits. The results, presented in Table 1, are all averaged over the set of 128 angles.

Table 1 also shows, for reference, the cost of a type (i) rotator using CSD coefficients, and the overall optimum cost, obtained by selecting the lowest cost rotator for each rotation angle. The average cost of each individual rotator type is also shown, along with the percentage of rotation angles for which that type achieved the minimum cost. The averages in Table 1 are over only those angles for which the chosen type has a solution of sufficient accuracy. Because of the limits imposed when constructing two-stage rotator options (vii), such rotators could not achieve accuracies of 12 or 15 bits for all angles, which is why the average cost shown in Table 1 is lower than the minimum; they never achieved 18-bit accuracy. RAG- $n$ was also not used for 18-bit accuracy, because the tables it requires, which grow rapidly in size with wordlength, had not been computed to sufficient wordlength. 
TABLE 1: Average adder-costs $\left(\mathrm{AC}_{\mathrm{AV}}\right)$ of rotators of accuracy $b=12,15$, and 18 bits, designed by different methods. $\%$ min is the percentage of cases in which the corresponding method achieved the minimum cost $\left(\mathrm{AC}_{\mathrm{MIN}}\right)$.

\begin{tabular}{|c|c|c|c|c|c|c|}
\hline Bits $b$ & 12 & 12 & 15 & 15 & 18 & 18 \\
\hline Type & $\mathrm{AC}_{\mathrm{AV}}$ & $\% \min$ & $\mathrm{AC}_{\mathrm{AV}}$ & $\% \min$ & $\mathrm{AC}_{\mathrm{AV}}$ & $\% \min$ \\
\hline (i) & 13.48 & 7 & 15.92 & 5 & 18.25 & 3 \\
\hline (ii) $\mathrm{BHM}$ & 12.47 & 18 & 14.93 & 9 & 17.24 & 6 \\
\hline (ii) RAG & 11.98 & 34 & 15.02 & 20 & - & 0 \\
\hline (iii) & 12.72 & 2 & 14.64 & 4 & 16.40 & 5 \\
\hline (iv) & 12.20 & 20 & 14.13 & 14 & 16.17 & 22 \\
\hline (v) & 12.22 & 16 & 14.06 & 17 & 16.07 & 24 \\
\hline (iii a) & 12.70 & 2 & 14.58 & 4 & 16.43 & 3 \\
\hline (iv a) & 12.17 & 24 & 14.09 & 17 & 16.18 & 23 \\
\hline (v a) & 13.19 & 23 & 14.02 & 20 & 16.09 & 26 \\
\hline (vi) & 11.22 & 49 & 12.98 & 56 & 14.73 & 66 \\
\hline (vii) & 10.07 & 30 & 10.43 & 9 & - & 0 \\
\hline (viii) & 11.57 & 38 & 13.92 & 31 & 16.09 & 23 \\
\hline (i) CSD & 15.36 & 0 & 19.25 & 0 & 23.00 & 0 \\
\hline $\mathrm{AC}_{\mathrm{MIN}}$ & 10.29 & - & 12.21 & - & 14.03 & - \\
\hline
\end{tabular}

It can be seen that the minimum cost is about two thirds of the cost of a conventional CSD implementation. No method is always optimum, which demonstrates the need to search all types. Of the individual types, type (vi) has the lowest average cost and the highest rate in achieving minimum cost, especially as the wordlength increases. Of the other types, type (viii) and type (ii) using RAG- $n$ perform well for 12-bit wordlength. Types (iv), (v), (iv a), and (v a) perform fairly well for all wordlengths.

\section{MULTIPLIERLESS FFT DESIGN}

One option for multiplierless implementation of the FFT is to replace the rotators in a conventional FFT structure by multiplierless rotators. Another option, for a radix-2 FFT, is to treat the butterflies as complex $2 \times 2$ matrix multiplications (equivalent to $4 \times 4$ real matrix multiplications) and apply CSE to them, or similarly, for a radix- $P$ FFT to treat the basic processing units (which consist of $P-1$ nontrivial rotators and a $P$-point FFT) as matrix multiplications. A third option is to implement the entire DFT as a matrix multiplication and apply CSE to it $[8] .{ }^{1}$

\subsection{FFT accuracy and output SNR}

Assume that all coefficients are quantised with $b$ bits after the binary point, and that the data wordlengths are sufficiently large so that the output noise due to requantisation (at "multiplier" outputs) is negligible. Then at the output of a radix-2 $\mathrm{N}$-point FFT, the ratio of the average output error variance due to coefficient quantisation to the output signal variance

\footnotetext{
tions.

${ }^{1}$ The author is grateful to an anonymous referee for these two sugges-
}

is given approximately by [19]

$$
\frac{\sigma_{E O}^{2}}{\sigma_{O}^{2}} \approx 2^{-2 b} \frac{\left(\log _{2} N\right)}{6}
$$

This formula (4) takes into account the fact that trivial rotations (i.e., those which rotate by integer multiples of $\pi / 2$ ) are computed with no error.

To characterise the accuracy of an FFT, it is therefore necessary to compute the effective wordlength $b$ of the nontrivial rotators. To do this, we first compute the RMS error of each nontrivial rotator in the FFT, and set $\sigma_{C}$ equal to the RMS of those errors, then use (3) to define an overall $\hat{b}$-bit accuracy, suitable for use in (4).

An alternative method of assessing accuracy of finiteprecision FFTs and DFTs [15] is to compute the Frobenius norm of the error between the effective DFT matrix, $\mathrm{F}_{\mathrm{E}}$, of the finite-precision transform and the exact DFT matrix, F, that is, the square root of the sum of absolute squares of the elements of $\mathbf{F}_{\mathrm{E}}-\mathbf{F}$.

\subsection{Optimisation approach}

The user must first define the transform size $N$ and the required accuracy.

For the approach in which the whole DFT is treated as a matrix multiplication, the DFT matrix elements are multiplied by an integer scale factor $k$ and rounded. The CSE algorithm from [9] is then applied to the result. As before, if an overall gain of unity is required, then $k$ is made a power of two (the required power of two can be deduced from the required accuracy). But if arbitrary gain is allowed, then a range of values of $k$ is searched to find the one which gives the required accuracy with lowest cost.

For the approaches in which the rotators (or butterflies or radix- $P$ units) in an FFT are replaced by multiplierless 
TABLE 2: Adder-cost (AC) and accuracy of DFTs designed by CSE methods from $[8,9]$. ( $N=$ FFT length; $b=$ bits after binary point; acc. $=$ accuracy (bits).)

\begin{tabular}{rrr|rrr}
\hline$N$ & $b$ & Acc. & \multicolumn{3}{|c}{ Adder-cost } \\
\hline 8 & 8 & 11.9 & 400 & 80 & 64 \\
8 & 16 & 18.5 & 528 & 84 & 68 \\
16 & 16 & 16.1 & 3296 & 364 & 220 \\
\hline
\end{tabular}

implementations, the user specifies the FFT radix and structure (e.g., mixed or split radix). The simplest optimisation method, which we call uniform (U-) scaling, is to apply the same scale factor $k$ to all paths through every stage of the FFT (apart from stages which contain only trivial rotations). For each value of $k$ in turn, the minimum-adder-cost rotators are found as described in Section 5, or the butterflies (or radix- $P$ units) are represented as fixed-point matrices and CSE is applied to them. If $k$ is not a power of 2, then each path with gain 1.0 or $\pm j$ in the unscaled FFT must be multiplied by $1.0 k$ or $\pm j k$ in the scaled FFT, and for this, the precomputed minimum-adder-cost solutions for individual real multiplications are used.

For rotator-based designs, it is only necessary to design rotators with rotation angles in the set $(0,1, \ldots, N / 8) \times 2 \pi / N$, because all the other required rotations are simple costless transformations of these [15].

For each value of $k$ in turn, the minimum-adder-cost and RMS accuracy of all the rotators are determined. Finally, the value of $k$ is determined which gives the minimum-cost solution that achieves at least the specified minimum-RMS accuracy.

The use of a common scale factor could give rise to a situation in which some rotators (or butterflies, etc.) are significantly more accurate than others, and so could be implemented with sufficient accuracy at lower cost. Therefore in a second method (called compatible (C-) scaling) the rotators are allowed to have different integer scale factors whose ratios are powers of 2 , so that subsequent binary shifts can be used to restore a single scale factor. In this method, the minimum costs and errors are first computed for each rotation angle separately, for each scale factor, and stored. Each scale factor $k$ in turn is then selected, and the stored results for all compatible scale factors (i.e., those equal to $k 2^{p} \leq k_{\max }$ for $p \geq 0$ ) are tested. The minimum-cost set in which all rotators have errors less than the specified limit is selected.

Only the two strategies described above were used for this paper. We also did not allow arbitrary phase rotations, as used in [1]. Alternative scaling strategies might produce further improvements; for example, a different scale factor could be allowed for each FFT stage.

\subsection{Results}

For the method in which butterfly units are treated as matrix multiplications, and CSE is applied, the resulting adder-cost
TABle 3: Adder-cost (AC) and accuracy of FFTs designed by the methods in this paper and [15]. $(N=$ FFT length; sc. $=$ scaling type; acc. $=$ accuracy (bits); FN = Frobenius norm of error (dB).)

\begin{tabular}{ccc|ccc|cc}
\hline \multirow{2}{*}{$N$} & \multirow{2}{*}{ Radix } & \multirow{2}{*}{ Sc. } & \multicolumn{3}{|c|}{ Our methods } & \multicolumn{2}{c}{ Methods in [15] } \\
& & AC & Acc. & FN & AC & FN \\
\hline \multirow{2}{*}{32} & 2 & U & 616 & 11.5 & -46 & 756 & -45 \\
& $2 / 4$ & U & 576 & 12.2 & -46 & - & - \\
\hline \multirow{2}{*}{128} & 2 & U & 4800 & 13.4 & -41 & 6727 & -41 \\
& $2 / 4$ & C & 3648 & 13.4 & -41 & - & - \\
\hline
\end{tabular}

was found to be always equal to that achieved by designing the corresponding rotator using CSE (i.e., rotator type (viii)) and adding four real adders to complete the butterfly. In the case of radix- 4 units, CSE applied to the whole radix- 4 unit required on average almost twice as many adders as the use of three type-(viii) rotators together with the 16 real adders required for a 4-point FFT. Therefore these options were not considered further.

The results obtained by applying CSE to the entire fixedpoint DFT matrix multiplication are shown in Table 2. Results are presented for 8- and 16-point DFTs with either 8 or 16 bits after the binary point, using the CSE methods in $[8,9]$. (Note that the results in $[8$, Table VIII] are only for part of the computation; Table 2 shows the total addercount using the method in [8].) It can be seen that the matrix CSE method in [9] gives lower adder-cost. These addercosts equal those achieved for FFTs based on multiplierless rotators (as can be seen from the corresponding entries in Table 5), but for the 16-point DFT the computation time of the CSE method was approximately 1000 times greater, and this ratio was found to increase exponentially with transform size and wordlength, making this method much less attractive for larger transforms.

For rotator-based FFTs, only power-of-2 (PO2) scale factors ever achieved minimum cost. This is because a significant number of the paths through each stage of an FFT have an unscaled gain of unity. For example, in radix-2 FFTs there are more unit gain paths than rotators, and in radix- 4 FFTs over a quarter of all paths have unit gain. If a non-PO2 scale factor is used, the adder-cost of multiplying every such path by that scale factor always outweighs any savings in the cost of the rotators.

The difference between U-scaling and C-scaling was small, but because the two scalings produce difference options for cost and accuracy, either can be slightly advantageous in any given case.

Table 3 presents results for designs to meet the specifications of the length-32 and - 128 radix-2 designs presented in [15]. It also shows the Frobenius norm of the error matrix, to allow comparison with [15]. The length-32 radix-2 FFT design using the methods of this paper requires 140 adders fewer than that in [15]. This is a reduction of $33 \%$ in the rotator adder-cost, but only an $18.5 \%$ reduction in the total adder-cost, because 320 adders are unavoidably used in the butterflies within the 32-point FFT. For the 128-point FFT, 


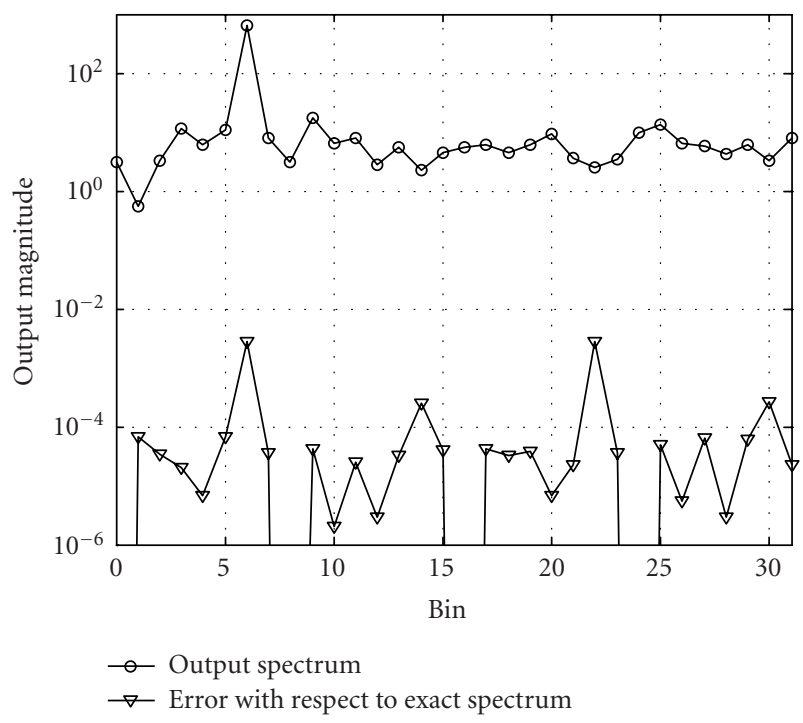

FIgURe 1: The 32-point Radix-2 FFT of single complex sinusoid in AWGN, using "16-bit-accuracy" coefficients.

TABle 4: Adder-cost (AC) and accuracy of an FFT designed by the methods in this paper and [1]. ( $N=$ FFT length; sc. = scaling type; acc. = accuracy (bits).)

\begin{tabular}{lcc|cc|cc}
\hline \multirow{2}{*}{$N$} & \multirow{2}{*}{ Radix } & \multirow{2}{*}{ Sc. } & \multicolumn{2}{|c|}{ Our methods } & \multicolumn{2}{|c}{ Methods in [1] } \\
& & AC & Acc. & AC & Acc. \\
\hline 16 & 4 & U & 224 & 16.1 & 352 & 13.0 \\
\hline
\end{tabular}

the new radix-2 design reduces the total adder-cost by $29 \%$ compared to [15]. These gains are due to both the search over a larger range of rotator structures and the fact that the coefficients are not constrained to coarsely-quantised SOPOT-1 values. If split-radix (2/4) FFTs are used, even lower cost designs are achieved, as shown in Table 3. The adder-cost saving compared to [15] increases to $24 \%$ for the 32 -point FFT, and $46 \%$ for the 128-point FFT.

Table 4 presents results for a design to meet the specifications of the length-16 radix-4 design presented in [1]. The design using the methods of this paper has three-bit greater accuracy than that in [1], with adder-cost reduced by $36 \%$, and unlike [1] it also has unity gain and no phase offset.

Table 5 presents results for transform sizes $N=8$ to 256 and target accuracies of 12,16 , and 20 bits. Radix- 2 results are presented for all sizes, and radix-4 results are given for $N=4^{K}$ only. Split-radix (2/4) designs are presented for $N>$ 16 (for $N=8$ or 16 the split-radix design has the same addercost as the radix- 2 or radix- 4 transform, resp.). In all other cases a split-radix (2/4) design gave the lowest cost, followed by a radix-4 design (for $N=4^{K}$ ), with radix-2 designs having the highest cost. The reduction in adder-cost compared to the use of CSD multipliers (in an FFT of the same size and radix) is shown in the final column of Table 5.

The reductions in adder-cost can be attributed to two factors-first, the effect of the number of rotators due to
TABle 5: Adder-cost (AC) and accuracy of various FFTs. $(N=$ FFT length; sc. $=$ scaling; acc. $=$ accuracy; redn. $=\%$ reduction in AC compared to CSD coefficients.)

\begin{tabular}{|c|c|c|c|c|c|}
\hline$N$ & Radix & Sc. & $\mathrm{AC}$ & Acc. bits & Redn.(\%) \\
\hline 8 & 2 & $\mathrm{U}$ & 64 & 11.9 & 6 \\
\hline 8 & 2 & $\mathrm{U}$ & 68 & 18.5 & 11 \\
\hline 8 & 2 & $\mathrm{U}$ & 72 & 26.0 & 22 \\
\hline \multirow{2}{*}{16} & 2 & $\mathrm{U}$ & 216 & 12.2 & 16 \\
\hline & 4 & $\mathrm{U}$ & 200 & 12.4 & 14 \\
\hline \multirow{2}{*}{16} & 2 & $\mathrm{U}$ & 240 & 16.1 & 15 \\
\hline & 4 & $\mathrm{U}$ & 220 & 16.0 & 14 \\
\hline \multirow{2}{*}{32} & 2 & $\mathrm{C}$ & 648 & 12.2 & 20 \\
\hline & $2 / 4$ & $\mathrm{U}$ & 576 & 12.2 & 18 \\
\hline \multirow{2}{*}{32} & 2 & $\mathrm{U}$ & 728 & 16.0 & 20 \\
\hline & $2 / 4$ & $\mathrm{U}$ & 636 & 16.1 & 19 \\
\hline \multirow{3}{*}{64} & 2 & $\mathrm{C}$ & 1684 & 12.0 & 25 \\
\hline & 4 & $\mathrm{U}$ & 1520 & 12.2 & 21 \\
\hline & $2 / 4$ & $\mathrm{C}$ & 1412 & 12.0 & 22 \\
\hline \multirow{3}{*}{64} & 2 & $\mathrm{U}$ & 1960 & 16.0 & 24 \\
\hline & 4 & $\mathrm{U}$ & 1696 & 16.0 & 24 \\
\hline & $2 / 4$ & $\mathrm{U}$ & 1604 & 16.0 & 24 \\
\hline \multirow{3}{*}{64} & 2 & $\mathrm{C}$ & 2212 & 20.54 & 27 \\
\hline & 4 & $\mathrm{C}$ & 1900 & 20.6 & 25 \\
\hline & $2 / 4$ & $\mathrm{C}$ & 1788 & 20.5 & 25 \\
\hline \multirow{2}{*}{128} & 2 & $\mathrm{C}$ & 4280 & 12.1 & 20 \\
\hline & $2 / 4$ & $\mathrm{C}$ & 3380 & 12.2 & 21 \\
\hline \multirow{2}{*}{128} & 2 & $\mathrm{U}$ & 4880 & 16.0 & 24 \\
\hline & $2 / 4$ & $\mathrm{U}$ & 3732 & 16.0 & 19 \\
\hline \multirow{3}{*}{256} & 2 & $\mathrm{C}$ & 10376 & 12.0 & 26 \\
\hline & 4 & $\mathrm{C}$ & 8888 & 12.0 & 24 \\
\hline & $2 / 4$ & $\mathrm{C}$ & 7604 & 12.1 & 18 \\
\hline \multirow{3}{*}{256} & 2 & $\mathrm{C}$ & 11840 & 16.0 & 28 \\
\hline & 4 & $\mathrm{U}$ & 10224 & 16.1 & 24 \\
\hline & $2 / 4$ & $\mathrm{C}$ & 8340 & 16.1 & 24 \\
\hline \multirow{3}{*}{256} & 2 & $\mathrm{C}$ & 13808 & 20.5 & 29 \\
\hline & 4 & $\mathrm{C}$ & 11592 & 20.5 & 27 \\
\hline & $2 / 4$ & $\mathrm{U}$ & 9448 & 20.5 & 26 \\
\hline
\end{tabular}

radix and structure choice, and secondly the result of using the cost-reduced rotators compared to CSD implementations. To determine the roles of these two factors, the average number of adders per rotator was calculated. Apart from sizes $N=8$ and 16 , the result was $9.75 \pm 0.5$ adders per rotator for 12-bit accuracy, $12.25 \pm 0.25$ for 16 bits, and $15 \pm 0.5$ for 20 bits. For $N=8$ and 16, the values are lower because the cost of rotation by a multiple of $\pi / 4$ is lower. Also the accuracies are higher than the number of bits after the binary point. This is because for $b=8$, the fixed-point approximation of $\sqrt{0.5}$ is $181 / 256$, which has an accuracy of 11.9 bits; while for $b=16$, the fixed-point approximation $46341 / 2^{16}$ has an accuracy of 18.5 bits.

To illustrate the overall performance of a typical multiplierless FFT, the "16-bit-" accuracy 32-point radix-2 FFT (as in 
Table 5) was used to compute the spectrum of the signal

$$
x(n)=20 \exp \left(\frac{j \pi n 6}{32}\right)+v(n), \quad n=1, \ldots, 32,
$$

where $v(n)$ is a unit-variance complex Gaussian noise. The computed spectrum, and the error between it and the exact spectrum, are shown in Figure 1. The measured signal-tonoise ratio was $104 \mathrm{~dB}$, in reasonable agreement with the value $97 \mathrm{~dB}$ given by (2) using $b=16.0$ bits.

\subsection{Discussion}

The resulting adder-count for the rotators is typically between one half and two thirds of the total adder-cost of the FFT, depending on the required accuracy.

However, these adder-costs may still not be the lowest that could be achieved. None of the published methods for reducing adder-cost guarantees optimality, except for the RAG- $n$ method [6] under certain circumstances. Further limitations of the search process described in this paper (such as the limited search of cascaded rotator implementations, the limited size of tables for optimum single multipliers, mentioned in Section 5, and the limited U- and C-scaling strategies) also mean that optimality is not claimed.

This paper concerns only minimisation of the addercount. In a VLSI implementation, it might also be desirable to limit the logic depth $[5,20]$ (which in this case implies limiting the number of adder delays in the rotator). This can be achieved by including the logic depth, with an appropriate weighting, into the "cost" measure throughout the process. In a similar way, a weighted cost for binary shifts could be included, if these were relevant to a particular implementation.

One way in which length- $M$ multiplierless FFTs could be used is as core blocks within a radix- $M$ FFT. Conventional multipliers could then be used for the twiddle factors between the radix- $M$ units.

Another option would be a completely parallel implementation of the FFT. For large transform sizes this would require large area, but it would be capable of extremely high processing throughput. Alternatively, if used to provide a more conventional throughput rate of FFTs per unit time, the circuit might be static (i.e., with no logic transitions occurring) for a large fraction of the time. In a CMOS implementation where power consumption is very low when a circuit is not changing its state, this might result in a low-power (though large area) implementation.

In some implementations the irregular structure of the split-radix transform is disadvantageous, but in a fully parallel implementation it would be of no disadvantage.

\section{CONCLUSIONS}

The methods described in this paper allow multiplierless rotators and multiplierless FFTs of arbitrary size and accuracy to be designed. We have shown that to minimise rotator adder-cost, it is necessary to consider every form of rotator described in Section 4. For FFTs, the most successful approach was based on the use of multiplierless rotators in conventional FFT structures. The application of matrix CSE methods to the fixed-point DFT multiplication did give equally good results for small transform sizes, but it took much longer time, and this computational disadvantage was found to increase rapidly with transform size and wordlength. For rotator-based FFT design it is only necessary to investigate PO2 scale factors. Searches are therefore fast.

The resulting adder-count for the rotators is typically between one half and two thirds of the total adder-cost of the FFT, for accuracies of 12-20 bits. For a given accuracy requirement, multiplierless FFTs designed using the methods in this paper have significantly lower adder-cost than previously described designs or implementations using conventional CSD coefficients.

As a result, fully or highly parallel VLSI implementations are feasible. Alternatively, the methods described in this paper could be used to design efficient length- $M$ FFTs for use in larger radix- $M$ transform processors.

\section{REFERENCES}

[1] A. M. Despain, "Very fast Fourier transform algorithms hardware for implementation," IEEE Trans. Comput., vol. 28, no. 5, pp. 333-341, 1979.

[2] A. G. Dempster and M. D. Macleod, "General algorithms for reduced-adder integer multiplier design," Electronics Letters, vol. 31, no. 21, pp. 1800-1802, 1995.

[3] A. G. Dempster and M. D. Macleod, "Constant integer multiplication using minimum adders," IEE Proceedings-Circuits, Devices and Systems, vol. 141, no. 5, pp. 407-413, 1994.

[4] O. Gustafsson, A. G. Dempster, and L. Wanhammar, "Extended results for minimum-adder constant integer multipliers," in Proc. IEEE International Symposium on Circuits and Systems (ISCAS '02), vol. 1, pp. 73-76, Scottsdale, Ariz, USA, May 2002.

[5] R. I. Hartley, "Sub-expression sharing in filters using canonic signed digit multipliers", IEEE Trans. Circuits Syst. II, vol. 43, no. 10, pp. 677-688, 1996.

[6] A. G. Dempster and M. D. Macleod, "Use of minimum-adder multiplier blocks in FIR digital filters," IEEE Trans. Circuits Syst. II, vol. 42, no. 9, pp. 569-577, 1995.

[7] M. Potkonjak, M. B. Srivastava, and A. Chandrakasan, "Multiple constant multiplications: efficient and versatile framework and algorithms for exploring common sub-expression elimination," IEEE Trans. Computer-Aided Design, vol. 15, no. 2, pp. 151-165, 1996.

[8] R. Pasko, P. Schaumont, V. Derudder, S. Vernalde, and D. Durackova, "A new algorithm for elimination of common sub-expressions," IEEE Trans. Computer-Aided Design, vol. 18, no. 1, pp. 58-68, 1999.

[9] M. D. Macleod and A. G. Dempster, "Common subexpression elimination algorithm for low-cost multiplierless implementation of matrix multipliers," Electronics Letters, vol. 40, no. 11, pp. 651-652, 2004.

[10] J. Liang and T. D. Tran, "Fast multiplierless approximations of the DCT with the lifting scheme," IEEE Trans. Signal Processing, vol. 49, no. 12, pp. 3032-3044, 2001.

[11] A. C. Zelinski, M. Püschel, S. Misra, and J. C. Hoe, "Automatic cost minimization for multiplierless implementations of discrete signal transforms," in Proc. IEEE International 
Conference on Acoustics, Speech, and Signal Processing (ICASSP '04), vol. 5, pp. 221-224, Montreal, Quebec, Canada, May 2004.

[12] M. D. Macleod, "Multiplierless Winograd and prime factor FFT implementation,” IEEE Signal Processing Lett., vol. 11, no. 9, pp. 740-743, 2004.

[13] P. Duhamel and H. Hollmann, "Split radix FFT algorithm," Electronics Letters, vol. 20, no. 1, pp. 14-16, 1984.

[14] W. A. Perera and P. J. W. Rayner, "Optimal design of discrete coefficient DFTs for spectral-analysis: extension to multiplierless FFTs," IEE Proceedings-G: Circuits, Devices and Systems, vol. 133, no. 1, pp. 8-18, 1986.

[15] S. C. Chan and P. M. Yiu, "An efficient multiplierless approximation of the fast Fourier transform using sum-of-powers-oftwo (SOPOT) coefficients," IEEE Signal Processing Lett., vol. 9, no. 10, pp. 322-325, 2002.

[16] M. D. Macleod and A. G. Dempster, "Multiplierless FIR filter design algorithms," IEEE Signal Processing Lett., vol. 12, no. 3, pp. 186-189, 2005.

[17] D. R. Bull and D. H. Horrocks, "Primitive operator digital filters," IEE Proceedings-G: Circuits, Devices and Systems, vol. 138, no. 3, pp. 401-412, 1991.

[18] A. M. Despain, "Fourier transform computers using CORDIC iterations," IEEE Trans. Comput., vol. 23, no. 10, pp. 993-1001, 1974.

[19] A. V. Oppenheim and C. J. Weinstein, "Effects of finite register length in digital filtering and the fast Fourier transform," Proc. IEEE, vol. 60, no. 8, pp. 957-976, 1972.

[20] A. G. Dempster, S. S. Dimirsoy, and I. Kale, "Designing multiplier blocks with low logic depth," in Proc. IEEE International Symposium on Circuits and Systems (ISCAS '02), vol. 5, pp. 773-776, Scottsdale, Ariz, USA, May 2002.

Malcolm D. Macleod was born in Cathcart, Glasgow, Scotland, in 1953. He was awarded the B.A. (with distinction) and M.A. degrees in electrical sciences, and the Ph.D. degree in digital signal processing, by the University of Cambridge, England, in 1974, 1978, and 1979, respectively. From 1978 to 1988 he worked for Cambridge Consultants Ltd. on a wide range of signal processing, electronics, and software projects. From 1988 to

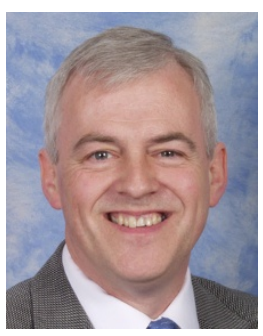

1995 he was a Lecturer in signal processing and communications in the Engineering Department of Cambridge University, and from 1995 to 2002 he was the Director of Research in the department. In 2002, he joined QinetiQ Ltd. as a Senior Research Scientist. He is a Fellow of the IEE (UK). He has published over 80 papers and conference papers, and contributed chapters to several books. His main research interests are in digital filter design, algorithms and architectures for DSP, nonlinear filtering, adaptive filtering, optimal detection, high-resolution spectrum estimation, beamforming and direction finding, and applications in sonar, radar, audio, instrumentation, and communication systems. 